\title{
Erratum to: A four-part working bibliography of neuroethics: Part 4 - Ethical issues in clinical and social applications of neuroscience
}

\author{
Kira Becker ${ }^{1}$, John R. Shook ${ }^{2}$, Martina Darragh ${ }^{3}$ and James Giordano ${ }^{4^{*}}$
}

After the publication of this article [1] it has come to our attention that the following quote from the article by Lombera and Illes [2] "...must have the power - defined by quality of knowledge and ease of access - to help shape that future.".. was missing a reference citation in the introduction.

The omission of the reference citation for this quote was the result of an error during the production process, and not an omission by, and/or error of the authors.

\footnotetext{
Author details

'Department of Neuroscience, Amherst College, Amherst, MA, USA.

${ }^{2}$ Department of Philosophy, University of Buffalo, Buffalo, NY, USA. ${ }^{3}$ Bioethics Research Library, Kennedy Institute of Ethics, Georgetown University, Washington, DC, USA. ${ }^{4}$ Neuroethics Studies Program, Pellegrino Center for Clinical Bioethics, and Department of Neurology, Georgetown University Medical Center, Washington, DC, USA.
}

Received: 12 June 2017 Accepted: 20 June 2017

Published online: 28 June 2017

\section{References}

1. Becker $\mathrm{K}$, et al. A four-part working bibliography of neuroethics: Part 4 - Ethical issues in clinical and social applications of neuroscience. Philosophy, Ethics, and Humanities in Medicine. 2017;12:1. doi:10.1186/s13010-017-0043-y.

2. Lombera S, Illes J. The International Dimensions of Neuroethics. Dev World Bioeth. 2009;9(2):57-64. doi:10.1111/j.1471-8847.2008.00235.x.

\footnotetext{
* Correspondence: james.giordano@georgetown.edu

${ }^{4}$ Neuroethics Studies Program, Pellegrino Center for Clinical Bioethics, and Department of Neurology, Georgetown University Medical Center, Washington, DC, USA
} 